(C2017, Elsevier. Licensed under the Creative Commons Attribution-NonCommercialNoDerivatives 4.0 International

http://creativecommons.org/about/downloads 


\title{
Aero-Optical Effects in Free and Wall-Bounded Turbulent Compressible Flows
}

\author{
V.N. Emelyanov ${ }^{1}$, I.V. Teterina ${ }^{1}$, K.N. Volkov $^{2, *}$, M.S. Yakovchuk ${ }^{1}$ \\ ${ }^{1}$ Baltic State Technical University, St Petersburg, Russia \\ ${ }^{2}$ Kingston University, London, United Kingdom
}

\begin{abstract}
The optical aberrations induced by turbulent compressible flows are a serious concern in airborne communication, imaging and optical systems because the quality of the laser beam degrades due to variations of the index of refraction along its path. The aero-optical aberrations produced by compressible flows are a direct consequence of the detailed physics of the flow. Time-accurate computational study of aero-optical distortions is performed based on large-eddy simulation technique because of its ability to account for the optical phase errors due to anisotropy of the flowfield and to resolve large-scale coherent structures at a reasonable computational cost. The results of numerical simulation of turbulent compressible flows in a flat plate boundary layer, free mixing layer and free jet provide general statistics of optical distortions by examining the time-averaged intensity pattern and instantaneous flowfield. The developed computational tools and results are beneficial in design and optimization of optical systems, where the control of laser beam propagation is an important problem, and coherent optical adaptive equipment and techniques based on the optical detection of light-scattering properties of the flow.
\end{abstract}

\section{Keywords}

Flight safety; Aero-optics; Light propagation; Turbulence; Large-eddy simulation; Vortex; Mixing layer; Free jet; Boundary layer

\section{Introduction}

The effects of hybrid (passive and active) flow control of the aero-optical and aerodynamic characteristics of the flow in the compressible free shear flows and boundary layers play an important role in flight safety. The aero-optical distortions induced by turbulent compressible flow are a serious concern of the airborne optical systems, because the quality of the beam degrades due to variations of the index of refraction along its path. As the index of refraction in air is proportional to its density, density gradients within the flowfield lead to a distortion of the wavefront as it passes through the flow. The performance of these systems is often limited by the interaction of the optical wavefront entering or exiting the aircraft with the turbulent flow surrounding the aircraft [1].

Amplitude and phase of the beam are subjected to fluctuations corresponding to the structural changes (broadening, deflection, splitting) of the beam [2]. When an initially planar optical wavefront passes a compressible flow or thermal environment, different parts of the wavefront experience different density of the medium and have different propagation

\footnotetext{
*Corresponding author: k.volkov@kingston.ac.uk
} 
speeds, and the wavefront becomes deformed (Figure 1). The consequences of such deformations include optical beam deflection (bore-sight error) and jitter, beam spread and loss of intensity. Wavefront distortions also cause reductions of resolution, contrast, effective range and sensitivity for optical sensors and imaging systems.

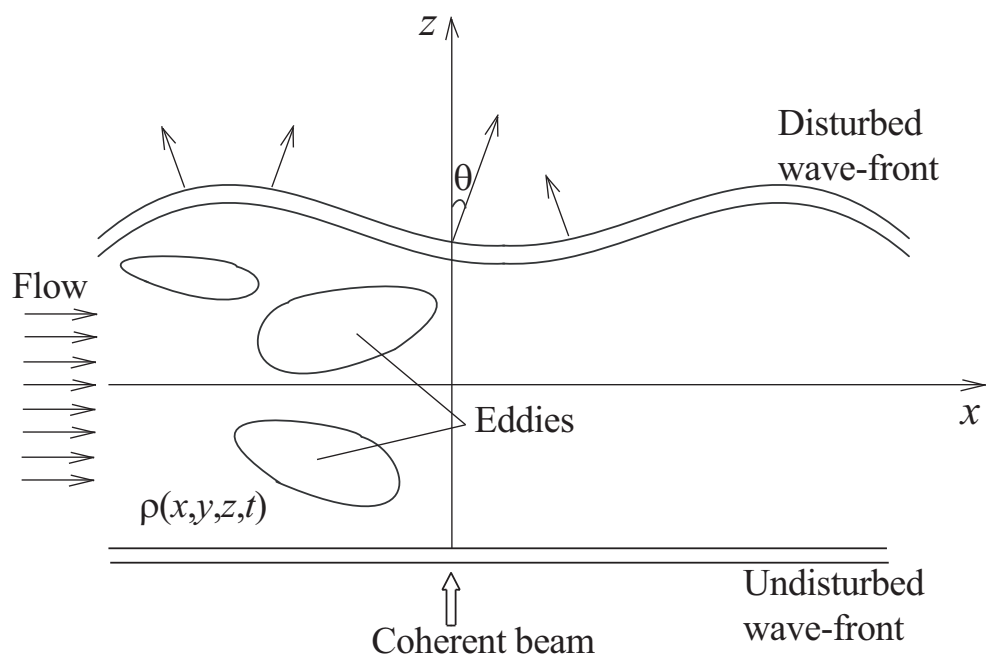

Figure 1. Propagation of coherent beam through a turbulent compressible flow

The recent developments in aero-optical research are summarized and reviewed in [2-4]. A comparison study of atmospheric propagation and aero-optics, which distinguished the aero-optical transmission from atmospheric propagation in terms of the optically distorting characteristic parameters of flowfields, is performed in $[5,6]$. The aero-optical interactions along the propagation path in turbulent compressible separated shear layers through experiments on direct imaging of the refractive index field are examined in [7]. To study the nature of optical aberrations, vortex models of the flow in the boundary layer and free mixing layer are considered, which are based on the exact solution of the plane Lamb vortex problem [8].

The key issue in modelling aero-optical effects is the choice of the method used to calculate the field of the fluctuating parameters of the turbulent flow, in particular, the field of density fluctuations $[2,9]$ (the density is related to the refractive index by the GladstoneDale law). A number of semi-empirical models of different levels of complexity have been developed for numerical simulations. The most popular models are the model of homogeneous turbulence [3] and the model of inhomogeneous turbulence [4]. In models [3,4,10], the dispersion of density fluctuations is taken as the difference between the medium densities near the wall and at the boundary layer edge. In the approach used in [11], the field of density fluctuations is related to temperature fluctuations using the equation of state (pressure fluctuations are neglected). The temperature fluctuations are found with the use of the Reynolds analogy and Prandtl mixing length model. A mathematical model of the optical window under the thermal environment and analysis of the optical path difference caused by the aero-optical effects are derived in [12].

The study [13] employs CFD results as the source data to discuss the problem of sparse measurement in aero-optics. For this purpose, proper orthogonal decomposition approach has been employed to reduce the amount of information required to compute or reconstruct the aberrated wavefront. A neural network model for high-speed turbulent boundary layer is proposed to solve the spatial frequency problem in sparse measurements. Decomposition of the instantaneous velocity and pressure fields into a time-averaged quantity, a random component that describes small-scale motion, and a periodic (coherent) component corre- 
sponding to large vortices, is used in [14].

Because of its ability to resolve large-scale motions at a reasonable computational cost, large-eddy simulation (LES) has recently been used in aero-optics. LES of a compressible turbulent mixing layer and ray tracing through it is performed in [15]. LES of aero-optical distortions in a fuselage/turret configuration is reported in $[16,17]$, where ray tracing is used to obtain the wavefront error and represented it in terms of Zernike polynomials. Instantaneous far-field intensity patterns using the Fraunhofer approximation is studied. A computational model for studying aero-optical imaging through the supersonic flowfields is presented in [18]. This model integrates the CFD grids with the model of angular spectrum propagation to construct serially connected optical sub-systems for representing the supersonic flowfields.

The aero-optical distortions produced by the largest-scale flow structures in the boundary layer on a flat plate, free mixing layer and free turbulent jet are simulated in this study. The semi-empirical correlations, analytical model and numerical model are developed to predict the dispersion of phase function. The results obtained are compared with available computational and experimental data.

\section{Phase function of wavefront}

The propagation of electromagnetic wave is described by the Maxwell equations. It is assumed that the time scale related to the wave propagation is much smaller than the turbulent time scale, the refractive index is time-independent, and the medium is non-conducting and has a constant magnetic susceptibility. Propagation of the wavefront is described by the equation

$$
\nabla^{2} \boldsymbol{E}-\frac{n^{2}}{c_{0}^{2}} \frac{\partial^{2} \boldsymbol{E}}{\partial t^{2}}=0
$$

where $\boldsymbol{E}$ is the electric field strength. The refractive index is defined as $n=c_{0} / c$, where $c$ and $c_{0}$ are the speeds of light in the medium and vacuum, respectively. For a monochromatic sinusoidal wave with angular frequency $\omega$, the equation (1) has an solution

$$
\boldsymbol{E}(\boldsymbol{r}, t)=\boldsymbol{E}_{0} \exp [\varphi(\boldsymbol{k}, \boldsymbol{r})-\omega t],
$$

where $\boldsymbol{E}$ is the field strength at point $\boldsymbol{r}$ at time moment $t$, and $\boldsymbol{E}_{0}$ is the field strength at the point $\boldsymbol{r}=0$. The wave number, $k$, angular frequency, $\omega$, and wavelength, $\lambda$, are coupled by the relationship $k=\omega c=2 \pi / \lambda$.

The angle describing the direction of the beam that passed through a test section of the variable-density medium is determined as the time derivative of the optical path of the beam

$$
\alpha(\boldsymbol{x}, t)=\frac{d \varphi(\boldsymbol{x}, t)}{d t},
$$

where $\varphi(\boldsymbol{x}, t)$ is the phase function of the wavefront. Integration over time gives

$$
\varphi(\boldsymbol{x})=\int_{0}^{t} \alpha(\boldsymbol{x}, t) d t .
$$


The main parameter of interest for practical applications is the difference between the instantaneous and mean values

$$
\sigma_{\varphi}(\boldsymbol{x})=\varphi(\boldsymbol{x})-\langle\varphi(\boldsymbol{x})\rangle .
$$

The transition from the time formulation of the problem to the space formulation is performed with the Taylor hypothesis of frozen turbulence.

The optical path length is determined by integrating the distribution of the refractive index along the beam propagation direction [3]

$$
\Delta \varphi(x, y, t)=k \int_{z_{1}}^{z_{2}} \Delta n(x, y, z, t) d z,
$$

where $\Delta n(x, y, z, t)$ is the variation of the refractive index along the beam propagation direction at time $t$, and $L=\left|z_{2}-z_{1}\right|$ is the thickness of test section. The density field is converted into the refractive index field. The dependence of the refractive index on fluid density is described by the Gladstone-Dale law [2]

$$
n(\boldsymbol{x})=1+G\left[\rho(\boldsymbol{x})-\rho_{\infty}\right],
$$

where $\rho_{\infty}$ is the free stream density and $G$ is the Gladstone-Dale constant. Gladstone-Dale constant is weakly dependent on the wavelength and is expressed by

$$
G(\lambda)=2.23 \times 10^{-4}\left(1+\frac{7.52 \cdot 10^{-3}}{\lambda^{2}}\right) \quad\left(\mathrm{m}^{3} / \mathrm{kg}\right),
$$

where $\lambda$ is wavelength in $\mu \mathrm{m}$.

\section{Dispersion of phase function}

Dispersion of small-scale fluctuations of density, $\sigma_{\rho}^{2}$, and the corresponding correlation length scale, $l_{\rho}$, are related to the dispersion of wave phase, $\sigma_{\varphi}^{2}$, by the expression [3]

$$
\sigma_{\varphi}^{2}=\alpha \beta^{2} \int_{0}^{L} \sigma_{\rho}^{2} l_{\rho} d y, \quad \beta=\frac{2 \pi}{\lambda} \frac{d n}{d \rho}=k G(\lambda),
$$

where $L$ is the optical path length, and the integral is taken across the boundary layer. The constant, $\alpha$, depends on the form of the correlation function assumed for the density fluctuations ( $\alpha=2$ for the exponential correlation function and $\alpha=\pi$ for the Gaussian correlation function). According to the equation (4), this leads to an $11 \%$ difference in the dispersion of phase fluctuations.

Length scale, $l_{\rho}$, is found by integrating the correlation function

$$
l_{\rho}=\int_{-\infty}^{+\infty} R_{\rho \rho}(y) d y
$$

In the conditions of local equilibrium, the correlation length scale coincides with the correlation scale of velocity fluctuations, $l_{\rho} \sim l_{u} \sim k^{3 / 2} / \varepsilon$, where $k$ is the turbulent kinetic energy 
and $\varepsilon$ is its dissipation rate. This assumption is not applicable near the wall, where velocity fluctuations turn to zero, therefore $k=0$ and $l_{u}=0$, but $l_{\rho} \neq 0$.

To estimate the phase fluctuations in the near-wall region, the equation (4) is usually replaced with a semi-empirical equation [3]

$$
\sigma_{\varphi}^{2}=\beta^{2} l_{y} \delta \sigma_{\rho}^{2},
$$

where $l_{y}$ is the integral turbulence length scale in the direction normal to the wall. It is assumed that $l_{y} \sim 0.1 \delta$, where $\delta$ is the boundary layer thickness. The dispersion of the density fluctuations is estimated as

$$
\sigma_{\rho}^{2}=A^{2}\left(\rho_{w}-\rho_{\infty}\right)^{2},
$$

where $\rho_{w}$ and $\rho_{\infty}$ are the density at the wall and the density in the free stream, and $A=0.1-$ 0.2 . At $l_{y} \ll \delta$, formula $(5)$ is refined by integrating across the boundary layer $[3,10]$

$$
\sigma_{\varphi}^{2}=\beta^{2} \int_{0}^{L} \sigma_{\rho}^{2}(y) l_{y}(y) d y .
$$

The models $[3,4,10]$ are based on equations (5) and (7), and the dispersion of density fluctuations is determined as the difference between the density near the wall (inner region) and the density in the outer region of the boundary layer. The applications of the models $[3,4]$ are discussed in [10]. In the models $[11,19]$, the density fluctuations are found by solving the transport equation of a passive scalar.

If pressure fluctuations are disregarded, density fluctuations are related to temperature fluctuations based on the equation of state $[11,19]$. The temperature fluctuations are determined using the Reynolds analogy between the velocity and temperature transport and the Prandtl mixing length model [20], whilst the accounting for the fact that the corresponding linear scales satisfy the relationship $l_{u} / l_{T}=\operatorname{Pr}_{t}$, where the turbulent Prandtl number is constant.

\section{Governing equations}

In Cartesian coordinates $(x, y, z)$, an unsteady three-dimensional compressible flow is described by the following equation

$$
\frac{\partial \boldsymbol{Q}}{\partial t}+\frac{\partial \boldsymbol{F}_{x}}{\partial x}+\frac{\partial \boldsymbol{F}_{y}}{\partial y}+\frac{\partial \boldsymbol{F}_{z}}{\partial z}=0 .
$$

A link between pressure and specific total energy has a form

$$
p=(\gamma-1) \rho\left[e-\frac{1}{2}\left(v_{x}^{2}+v_{y}^{2}+v_{z}^{2}\right)\right] .
$$

The vector of conservative variables, $\boldsymbol{Q}$, and the flux vectors, $\boldsymbol{F}_{x}, \boldsymbol{F}_{y}$ and $\boldsymbol{F}_{z}$, have the following form

$$
\boldsymbol{Q}=\left(\begin{array}{c}
\rho \\
\rho v_{x} \\
\rho v_{y} \\
\rho v_{z} \\
\rho e
\end{array}\right)
$$




$$
\begin{gathered}
\boldsymbol{F}_{x}=\left(\begin{array}{c}
\rho v_{x} \\
\rho v_{x} v_{x}+p-\tau_{x x} \\
\rho v_{x} v_{y}-\tau_{x y} \\
\rho v_{x} v_{z}-\tau_{x z} \\
(\rho e+p) v_{x}-v_{x} \tau_{x x}-v_{y} \tau_{x y}-v_{z} \tau_{x z}+q_{x}
\end{array}\right), \\
\boldsymbol{F}_{y}=\left(\begin{array}{c}
\rho v_{y} \\
\rho v_{y} v_{x}-\tau_{y x} \\
\rho v_{y} v_{y}+p-\tau_{y y} \\
\rho v_{y} v_{z}-\tau_{y z} \\
(\rho e+p) v_{y}-v_{x} \tau_{y x}-v_{y} \tau_{y y}-v_{z} \tau_{y z}+q_{y}
\end{array}\right), \\
\boldsymbol{F}_{z}=\left(\begin{array}{c}
\rho v_{z} \\
\rho v_{z} v_{x}-\tau_{z x} \\
\rho v_{z} v_{y}-\tau_{z y} \\
\rho v_{z} v_{z}+p-\tau_{z z} \\
(\rho e+p) v_{z}-v_{x} \tau_{z x}-v_{y} \tau_{z y}-v_{z} \tau_{z z}+q_{z}
\end{array}\right)
\end{gathered}
$$

The components of viscous stress tensor and components of heat flux vector are found from the relations

$$
\tau_{i j}=\mu_{e}\left(\frac{\partial v_{i}}{\partial x_{j}}+\frac{\partial v_{j}}{\partial x_{i}}-\frac{2}{3} \frac{\partial v_{k}}{\partial x_{k}} \delta_{i j}\right), \quad q_{i}=-\lambda_{e} \frac{\partial T}{\partial x_{i}} .
$$

Here, $t$ is the time, $\rho$ is the density, $v_{x}, v_{y}$, and $v_{z}$ are the velocity components in the coordinate directions $x, y$, and $z, p$ is the pressure, $e$ is the total energy per unit mass, $T$ is the temperature, and $\gamma$ is the specific heat ratio.

The equation (8) is suitable for both laminar and turbulent flows. The effective viscosity, $\mu_{e}$, is calculated as a sum of molecular viscosity, $\mu$, and eddy viscosity, $\mu_{s}$, and the effective thermal conductivity, $\lambda_{e}$, is expressed in terms of viscosity and Prandtl number

$$
\mu_{e}=\mu+\mu_{s}, \quad \lambda_{e}=c_{p}\left(\frac{\mu}{\operatorname{Pr}}+\frac{\mu_{s}}{\operatorname{Pr}_{s}}\right)
$$

where $c_{p}$ is the specific heat capacity at constant pressure. Molecular Prandtl number and sub-grid Prandtl number are $\operatorname{Pr}=0.72$ and $\operatorname{Pr}_{s}=0.9$ for air. The Sutherland's law is used to obtain molecular viscosity as a function of temperature

$$
\frac{\mu}{\mu_{*}}=\left(\frac{T}{T_{*}}\right)^{3 / 2} \frac{T_{*}+S_{0}}{T+S_{0}},
$$

where $\mu_{*}=1.68 \times 10^{-5} \mathrm{~kg} /(\mathrm{m} \mathrm{s}), T_{*}=273 \mathrm{~K}$ and $S_{0}=110.5 \mathrm{~K}$ for air.

In the Smagorinsky sub-grid scale model, the eddy viscosity is calculated as

$$
\mu_{s}=\rho\left(C_{S} \Delta\right)^{2}|S|
$$

where

$$
|S|=\left(2 S_{i j} S_{i j}\right)^{1 / 2}, \quad S_{i j}=\frac{1}{2}\left(\frac{\partial v_{i}}{\partial x_{j}}+\frac{\partial v_{j}}{\partial x_{i}}\right)
$$


It is usually assumed that $C_{S} \sim 0.1$.

To take into account the influence of the wall on the mixing length, $C_{S} \Delta$, the equation (9) is supplemented by the Van Driest damping function

$$
f_{\mu}=1-\exp \left[-\left(\frac{y^{+}}{25}\right)^{3}\right]
$$

where $y^{+}=\rho u_{\tau} y / \mu$ is the non-dimensional near wall coordinate, $u_{\tau}=\left(\tau_{w} / \rho\right)^{1 / 2}$ is the dynamic velocity, and $\tau_{w}$ is the wall shear stress.

The filter width, $\Delta$, is related to the control volume of the mesh

$$
\Delta=V^{1 / 3}=(\Delta x \Delta y \Delta z)^{1 / 3},
$$

where $V$ is the volume of cell, $\Delta x, \Delta y$, and $\Delta z$ are mesh steps in the coordinate directions $x, y$, and $z$, respectively. In the boundary layer, the mesh step in the direction normal to the wall, $\Delta x$, is replaced by the $\widehat{\Delta} x$, and the filter width is found as

$$
\Delta=(\widehat{\Delta} x \Delta y \Delta z)^{1 / 3}
$$

The modified representation of the filter width gives $\widehat{\Delta} x=\Delta x$ near the wall, and $\widehat{\Delta} x=\bar{\Delta} x$ far from the wall. The mesh step size, $\Delta x$, smoothly changes between the thresholds. The modified mesh step size, $\bar{\Delta} x$, is the averaged of $\Delta x$ in the near-wall region, and the modified mesh step size, $\widehat{\Delta} x$, is found from the formula

$$
\widehat{\Delta} x=\left[\left(\frac{1}{\Delta x}\right)^{m}+\left(\frac{1}{\bar{\Delta} x}\right)^{m}\right]^{-1 / m},
$$

where $m=3$. A smaller width of the filter allows reproduction of a wider frequency range of fluctuations of flow parameters, whereas an increase in filter width facilitates smoothing of the solution.

\section{$5 \quad$ Numerical method}

Numerical calculations are performed with unstructured in-house finite volume CFD code [21]. The code uses an edge-based data structure to give the flexibility to run on meshes composed of a variety of cell types. The fluxes are calculated on the basis of flow variables at nodes at either end of an edge, and an area associated with that edge (edge weight). The edge weights are pre-computed and take into account the geometry of the cell. The non-linear CFD solver works in an explicit time marching fashion, based on a three-step Runge-Kutta stepping procedure and piecewise parabolic method. The governing equations are solved with Chakravarthy-Osher scheme for inviscid fluxes and the central difference scheme of the 2nd order for viscous fluxes. Convergence to a steady state is accelerated by the use of multigrid techniques, and by the application of block-Jacobi preconditioning for high-speed flows, with a separate low-Mach number preconditioning method for use with low-speed flows. The sequence of meshes is created using an edge-collapsing algorithm.

Accumulation of numerical errors in simulations was controlled based on the algorithm described in [22]. The computational procedure is implemented in $\mathrm{C} / \mathrm{C}++$ programming language. Parallelization of the computational procedure is performed by a message passing interface (MPI) and general-purpose graphics processing units (GPU) [23]. 


\section{Results and discussion}

The results of numerical simulations of turbulent flows in a flat plate boundary layer, free mixing layer and free round turbulent jet provide general statistics of optical distortions by examining the time-averaged intensity patterns and instantaneous flowfield.

\subsection{Boundary layer on a flat plate}

The computations are performed at $\operatorname{Re}_{\delta}=2.6 \times 10^{4}$ (Reynolds number based on the boundary layer thickness, $\delta=18 \mathrm{~mm}$ ) and $\operatorname{Re}_{\delta_{m}}=2.8 \times 10^{3}$ (Reynolds number based on the momentum thickness, $\delta_{m}=2.2 \mathrm{~mm}$ ). The mesh contains $180 \times 90 \times 90$ cells. For this mesh, $x^{+}=45$, $y_{\min }^{+}=1$ and $z^{+}=14$. The time step is $\Delta t=2.1 \times 10^{-5} \mathrm{~s}$, and 40,000 time steps are performed to obtain time-averaged pattern of the flow.

No-slip and no-penetration boundary conditions are specified on the wall. The plate has a constant temperature $T_{w}=300 \mathrm{~K}$. Non-reflecting boundary conditions are specified on the boundaries through which the gas leaves the computational domain. Periodic boundary conditions are applied in spanwise direction.

The velocity profile in the near-wall region of the boundary layer based on LES calculations is in a good agreement with the Reinhardt law [24] based on the experimental data and covering the viscous sub-layer, buffer region and logarithmic region of the boundary layer (Figure 2).

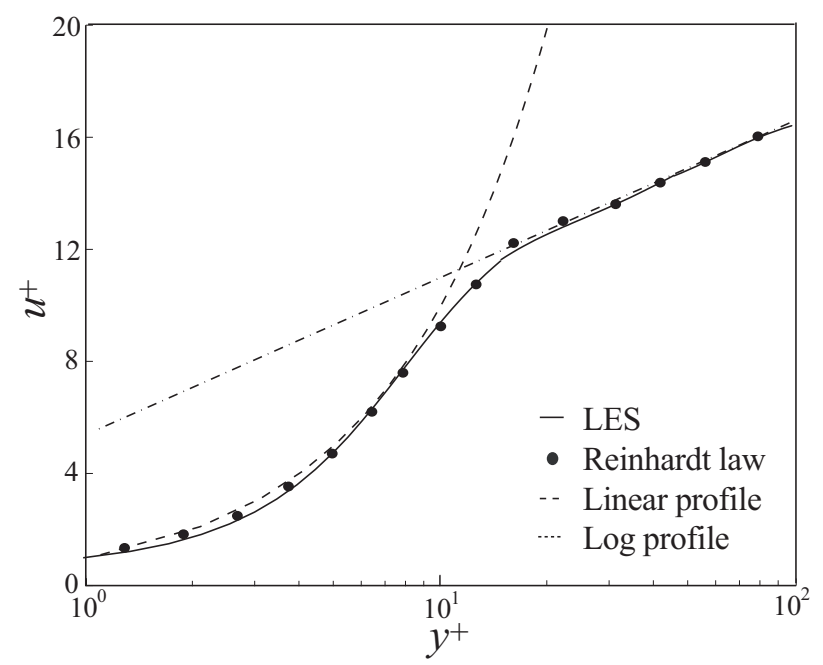

Figure 2. Velocity profile in the boundary layer

The resulting density fluctuations are by 10-20\% higher than the experimental values, presented in [10] at $\mathrm{M}=0.88$ (Figure 3). The maximum of the $y$ axis distribution of the density fluctuations is located well close to the wall. At $0.2<y / \delta<0.6$, the root-meansquare density remains nearly constant, and the dispersion of phase fluctuations is uniform in this region.

Comparison of the length scales $l_{u}$ and $l_{\rho}$ shows that they are equal only in the interval $0.1<y / \delta<0.22$. In the remaining part of the boundary layer, the correlation scale of the velocity fluctuations is smaller than that of the density fluctuations.

Using the vortex model [8] and assuming that the beam passes through the vortex center, integrating from $-\infty$ to $+\infty$ gives the following relation

$$
\Delta \varphi=-2 \pi \frac{G}{\gamma}\left(\frac{U}{a_{\infty}}\right)^{2} \rho_{0} R .
$$




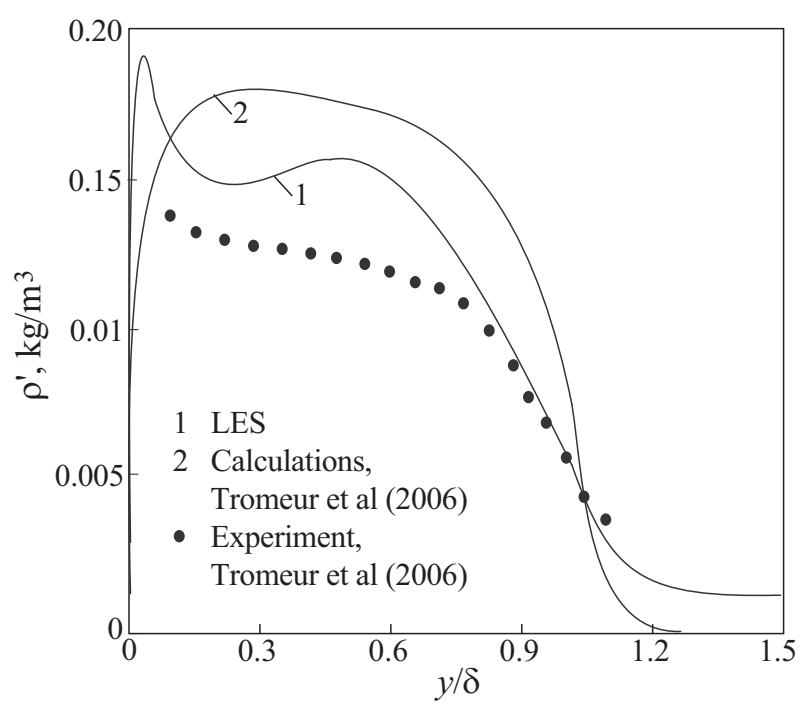

Figure 3. Density fluctuations in the boundary layer

The subscript 0 refers to parameters at the stagnation point.

An uniform chain of vortices located on one line and moving with a constant velocity is considered. Each vortex inserts optical perturbations whose maximum intensity is determined in accordance with the equation (10). Assuming these perturbations to have an approximately sinusoidal character, the dependence of the intensity of optical aberrations on the vortex density, velocity, intensity and vortex radius is obtained

$$
\sigma_{\varphi} \approx 4.48 G \theta^{2} \mathrm{M}_{\infty}^{2} \rho_{0} R
$$

where $\theta=U / U_{\infty}$ is the turbulence intensity, $\mathrm{M}_{\infty}$ is the free stream Mach number. Under the assumption that $R \sim \delta^{*} / 2$, where $\delta^{*}$ is the momentum thickness, and that the turbulence intensity is $10 \%$, the equation (11) yields

$$
\sigma_{\varphi} \approx 1.48 \times 10^{-5} \Phi
$$

where $\Phi=\delta^{*} \rho_{0} \mathrm{M}_{\infty}^{2} / \rho_{\infty}$. According to the experimental data [25], the constant factor in the equation (12) is $2.4 \times 10^{-5}$. The equation (12) yields a linear dependence of the optical path length of the beam on dynamic pressure, which agrees with the data of [26], but contradicts the data of [27], which state that $\sigma_{\varphi} \sim p_{0}^{1 / 2}$.

The results of calculations concerning the boundary layer on a flat plate are shown in the Figure 4 (it is assumed that $\alpha=2$ ). The dispersions predicted with the equation (7), developed in [10] (line 4), are overestimated as compared to those calculated with the equation (5) at $A=0.1$ and $A=0.2$, developed in [3] (line 2 and line 3). Unlike the predictions based on correlation, developed in [10] (line 4), the dispersion of the wavefront phase function provided by LES calculations (line 1) has an inflection point.

Figure 5 shows the results calculated for the boundary layer on a flat plate by the equation (12) (solid line), data of [25] (symbols $\circ$ and $\square$ ) and LES results for $x=0.11$ (symbols •) and 0.16 (symbols $\mathbf{\square}$ ). It is seen that numerical calculations yield lower values of the dispersion of the wavefront phase function than the results obtained in [25] for both values of $x$ coordinate. 


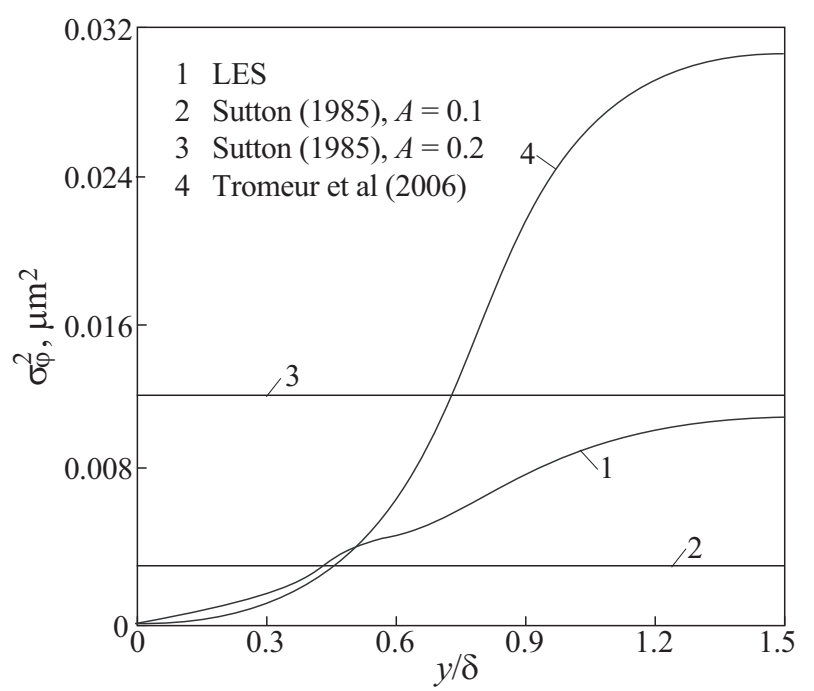

Figure 4. Dispersion of phase fluctuations in the boundary layer

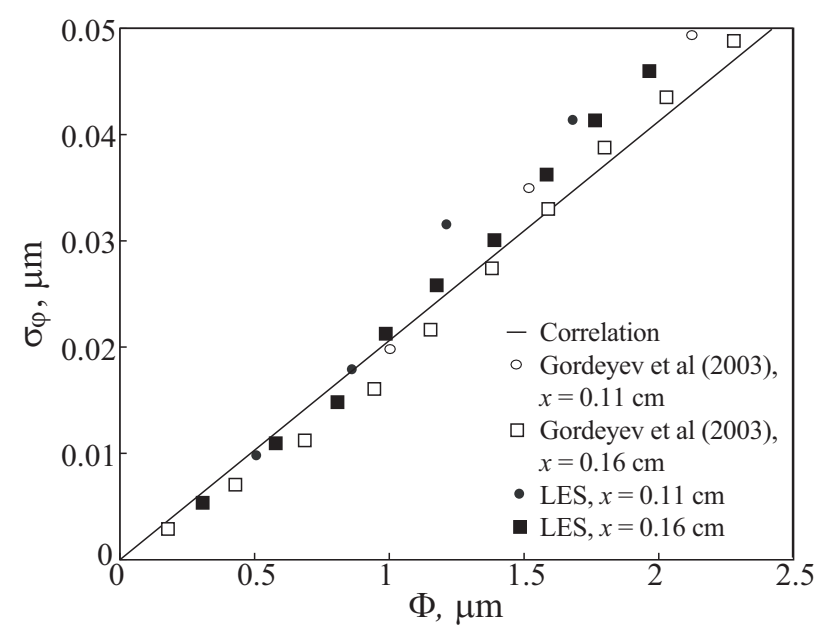

Figure 5. Root-mean-square values of optical aberrations in the boundary layer

\subsection{Free mixing layer}

Two semi-infinite gas flows move in one direction with velocities $u_{1}$ and $u_{2}$ along the plane $x<0, y=0$ (Figure 6). The flows contact each other at the point $x=0$ and further on (with $x>0$ ) the boundary between them is turbulised.

The flow in the free mixing layer is characterized by the convective Mach number

$$
\mathrm{M}_{c}=\frac{u_{1}-u_{2}}{a_{1}+a_{2}} .
$$

Momentum thickness is

$$
\delta_{m}(x)=\frac{1}{(\Delta u)^{2}} \int_{-\infty}^{+\infty} \rho\left(u-u_{1}\right)\left(u_{2}-u\right) d y .
$$

Reynolds number based on velocity difference, Reynolds number based on convective velocity and Reynolds number based on vorticity thickness are as follows

$$
\operatorname{Re}_{m}=\frac{\Delta u \delta_{m 0}}{\nu}, \quad \operatorname{Re}_{c}=\frac{u_{c} \delta_{m 0}}{\nu}, \quad \operatorname{Re}_{\omega}=\frac{\Delta u \delta_{\omega 0}}{\nu},
$$




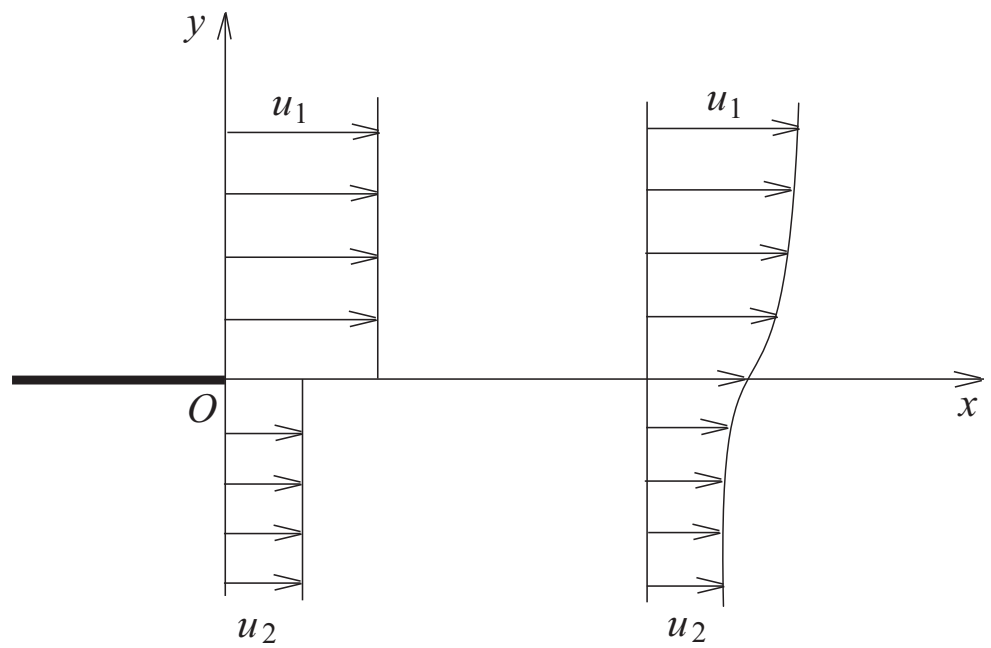

Figure 6. Free mixing layer

where $\delta_{\omega 0}$ is the vorticity thickness at $x=0$. Vorticity thickness is

$$
\delta_{\omega}(x)=\frac{u_{\max }-u_{\min }}{\max _{y}(\partial u / \partial y)} .
$$

The velocities of mixing flows are fixed at $u_{1}=78 \mathrm{~m} / \mathrm{s}$ and $u_{2}=200 \mathrm{~m} / \mathrm{s}$. The working fluid is air. For these parameters, $\delta_{\omega 0}=0.021 \mathrm{~m}$ and $\mathrm{Re}_{\omega}=21600$, which correspond to fully developed turbulent flow. The computations are performed for $\operatorname{Re}_{m}=2 \times 10^{5}$ within the range $\mathrm{M}_{c}=0.15-0.8$. At $\mathrm{M}_{c}=0.15$, compressibility has no effect on the properties of the flow $\left(\rho_{2} / \rho_{1}=1\right)$, while at $\mathrm{M}_{c}=0.9$ the density ratio is $\rho_{2} / \rho_{1}=4$.

The computational domain has a length $25 \delta_{\omega 0}$ and width $6 \delta_{\omega 0}$ (the $y$ coordinate varies in the interval $\left.-3 \delta_{\omega 0}<y<+3 \delta_{\omega 0}\right)$. The thickness of the computational domain is $5 \delta_{\omega 0}$. The mesh consists of $250 \times 80 \times 80$ cells. The mesh is refined to the edge of the plate and near the flow separation plane. The time step is $\Delta t=1.5 \times 10^{-3} \mathrm{~s}$, and 50,000 time steps are performed to obtain time-averaged pattern of flowfield.

Free shear flows are unstable, and oscillation in these flows arises in the absence of external sources of disturbances. The profile of streamwise velocity is defined as [28]

$$
u(y)=\frac{1}{2}\left(u_{1}+u_{2}\right)+\frac{1}{2}\left(u_{1}-u_{2}\right) \tanh \left[\frac{y}{2 \delta_{m}(0)}\right] \zeta, \quad \delta_{m}(0)=\frac{\Delta u}{(d u / d y)_{y=0}},
$$

where $\delta_{m}(0)$ is momentum thickness at the point $x=0$, and $\zeta$ is a random number chosen from the normal distribution of probability with a zero mathematical expectation and a unit variance.

Non-reflecting boundary conditions are applied to the outlet boundary. Slip boundary conditions are specified on the remote boundaries of fluid domain in $y$ direction. Periodic boundary conditions are applied in $z$ direction.

Momentum thickness decreases with increasing convective Mach number as shown in the Figure 7. Symbols • correspond to the computational data, symbols $\square$ correspond to data of [29], symbols $\Delta, \nabla$ and $\diamond$ correspond to data of [30], and symbols $\circ$ correspond to data of [31].

The profiles of the density fluctuations in the mixing layer are shown in the Figure 8 at $\mathrm{M}_{c}=0.8$. The distributions of the density and pressure fluctuations in the cross sections of the mixing layer are similar with a maximum at the line separating the mixing flows. The 


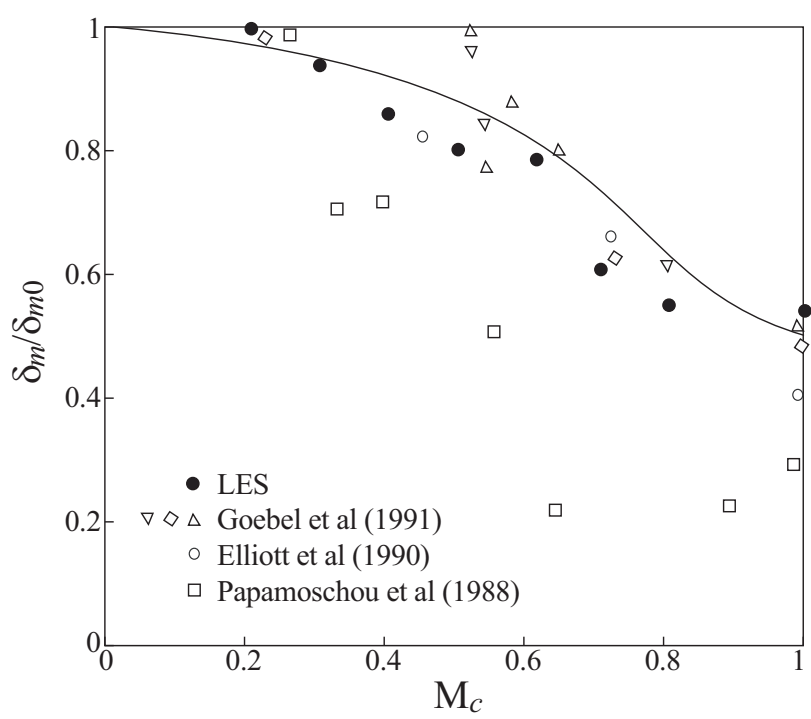

Figure 7. Dependence of momentum thickness on convective Mach number

profiles of pressure fluctuations are filled to a greater extent, and the maximal amplitude of the pressure fluctuations is close to a linear dependence on the streamwise coordinate. Small deviations from the linear dependence occur only at $x / L>0.68$.

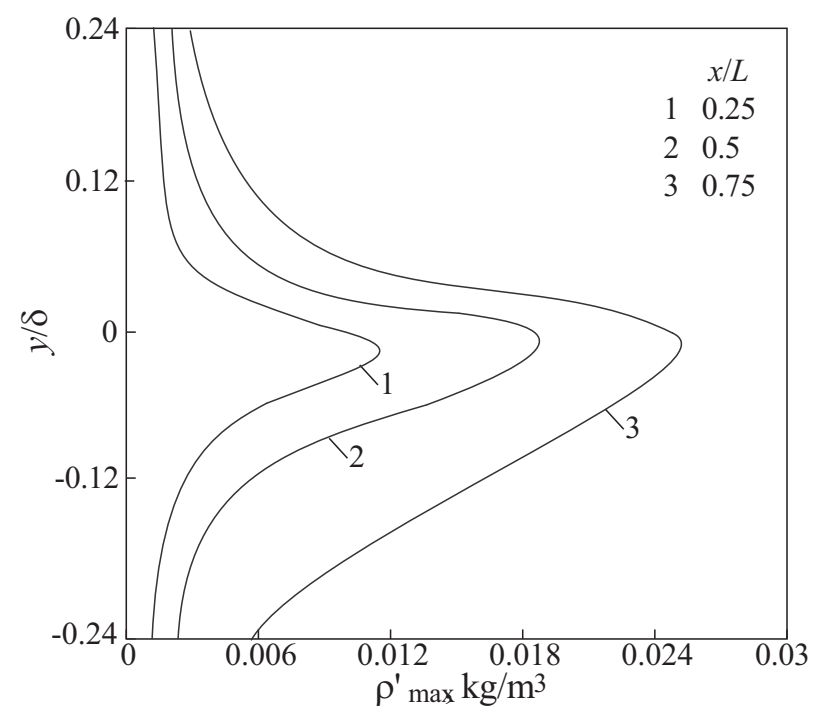

Figure 8. Density fluctuations in the cross section of the mixing layer

For isentropic conditions, the change in density is directly proportional to the change in pressure and inversely proportional to the change in temperature. Assuming that the change in pressure in the mixing layer is approximately proportional to the squared characteristic velocity $U_{c}=\left(u_{2}+u_{1}\right) / 2$, the density ratio is

$$
\frac{\Delta \rho}{\rho_{0}}=\frac{1}{\gamma} \frac{\Delta p}{p_{0}} \approx \frac{1}{\gamma} \frac{\rho_{0}}{p_{0}} U_{c}^{2}=\mathrm{M}_{c}^{2} .
$$

The root-mean-square value of the optical aberrations is related to the integral of the change in density. Choosing the vorticity thickness as a characteristic scale, the dispersion is found from

$$
\sigma_{\varphi} \approx G \Delta \rho \delta_{\omega} \approx G \rho_{0} \mathrm{M}_{c}^{2} \delta_{\omega} .
$$


In equation (13), the vorticity thickness is defined as [29]

$$
\frac{d \delta_{\omega}}{d x}=0.17 \frac{(1-\lambda)\left(1+s^{1 / 2}\right)}{1+\lambda s^{1 / 2}}
$$

where $\lambda=u_{2} / u_{1}, s=\rho_{2} / \rho_{1}$. Taking into account that $\delta_{\omega} \sim x$, a linear dependence of the optical aberrations on the coordinate, $\sigma_{\varphi} \sim \rho_{0} \mathrm{M}_{c}^{2} x$, is obtained.

Phase perturbations in the case of the wave passing through a layer of thickness $L=$ $\left|z_{2}-z_{1}\right|$ are found by integrating the distribution of the refractive index over the layer thickness [9]

$$
\widetilde{\varphi}(x, y, t)=\frac{\varphi(x, y, t)}{k_{0} L \Delta n} \simeq \frac{1}{L \Delta n} \int_{z_{1}}^{z_{2}}\left[n(x, y, z, t)-n_{\infty}\right] d z
$$

It follows from the equation (14) that the following relation is valid in the direction of beam propagation

$$
\psi(x)=L \frac{\partial \widetilde{\varphi}}{\partial x}=\frac{1}{\Delta n} \int_{z_{1}}^{z_{2}} \frac{\partial n}{\partial x} d z
$$

where $L$ is the size of the turbulent mixing zone. The spectrum follows the dependence

$$
S_{\psi}\left(k_{x} L\right) \sim\left(k_{x} L\right)^{2} S_{\varphi}\left(k_{x} L\right) .
$$

For the mixing layer, it is assumed that $\Delta n=\left|n_{1}-n_{2}\right|$ and $L=2 \delta$, where $\delta$ is the local width of the mixing zone. The subscripts 1 and 2 refer to the mixing flows.

The results presented in the Figure 9 are calculated for the mixing layer by the semiempirical dependence $(13)$ at $\mathrm{M}_{c}=0.24\left(u_{1}=260 \mathrm{~m} / \mathrm{s}\right.$ and $\mathrm{M}_{1}=0.77, u_{2}=0.06 \mathrm{~m} / \mathrm{s}$ and $\mathrm{M}_{2}=0.06$, in this case $\left.d \delta_{\omega} / d x \approx 0.25\right)$ are in reasonable agreement with the data of the physical experiment [5] (symbols o correspond to $A=20 \mathrm{~cm}$, and symbols $\mathbf{\square}$ correspond to $A=30 \mathrm{~cm})$ in the case of rather large aperture sizes $(A>20 \mathrm{~cm})$ and with LES results (symbols •).

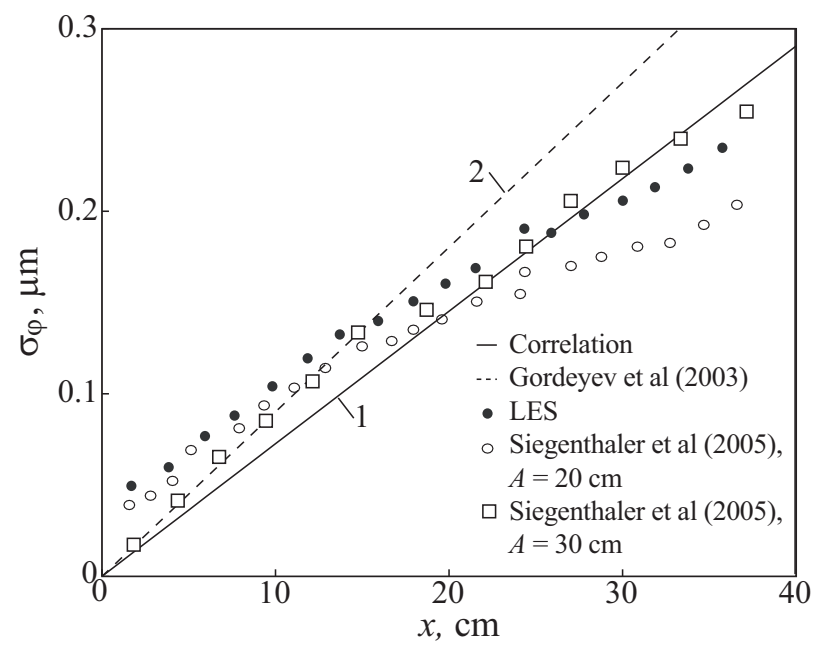

Figure 9. Root-mean-square values of optical aberrations in the mixing layer

Dashed line in the Figure 10 shows the results obtained in [25] with the use of a sinusoidal law for the deflection angle $\alpha=\sin (2 \pi f t)$, where $f=U_{c} / \Lambda$ is the frequency of vortex 
formation, $\Lambda$ is the vortex size and $U_{c}$ is the characteristic velocity. Such an approach allows obtaining the final dependence of the magnitude of optical aberrations on the streamwise coordinate. The agreement between the predicted and measured data, however, becomes worse in the downstream direction.

The spectrum is shown in the Figure 10. The compressibility only has a weak influence on the spectrum behavior (this influence is largely displayed at large wave numbers).

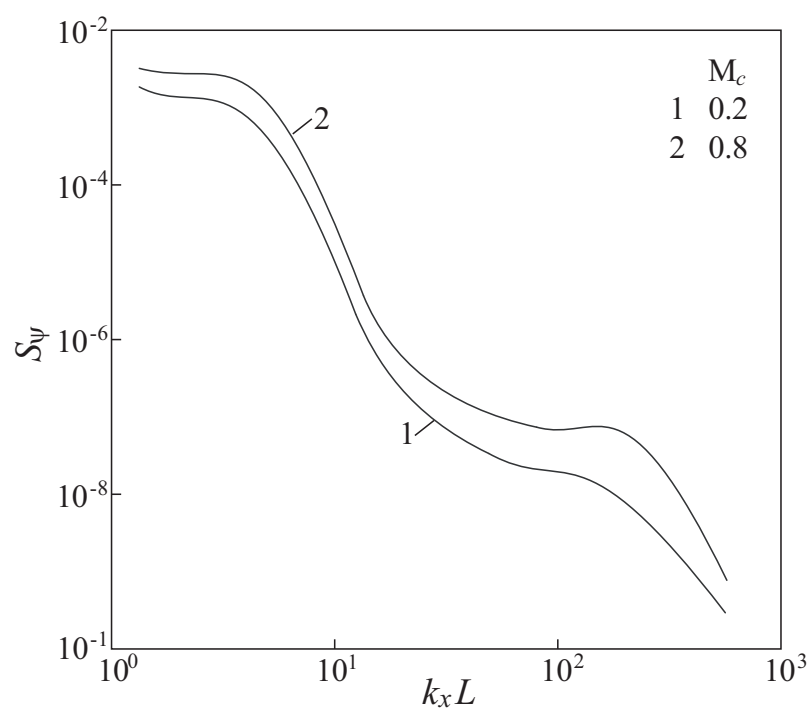

Figure 10. Spectrum of phase fluctuations in the free mixing layer

\subsection{Free turbulent jet}

A free turbulent jet is considered, which flows out of a round nozzle into submerged space. The origin of point is located at the nozzle outlet. The positive reading of $x$ coordinate is made in the direction of propagation of the jet. The radius of nozzle, $r_{a}$, is the characteristic scale for variables with the dimensionality of length. The velocity $u_{a}$ and temperature $T_{a}$ at the nozzle outlet are the characteristic scales for variables with the dimensionality of velocity and temperature. The temperature of surrounding fluid is $T_{\infty}$. The flow in the jet is characterized by the preheating parameter, $\vartheta=T_{a} / T_{\infty}$, and the intensity of turbulence, $\theta=u^{\prime} / u_{a}$, where $u^{\prime}$ is the fluctuation velocity.

The calculations are performed for $r_{a}=5 \mathrm{~mm}, u_{a}=80 \mathrm{~m} / \mathrm{s}, \rho_{a}=0.58-1.26 \mathrm{~kg} / \mathrm{m}^{3}$, $T_{a}=280-600 \mathrm{~K}, \rho_{\infty}=0.58-1.26 \mathrm{~kg} / \mathrm{m}^{3}, T_{\infty}=280-600 \mathrm{~K}$. The parameters at the nozzle outlet correspond to Reynolds number $\mathrm{Re}=1.2 \times 10^{5}$, which is maintained constant with relevant variation of dynamic viscosity, and to the range of variation of preheating parameter $\vartheta=0.48-2.15$.

The calculations are performed in the domain $\left[0, L_{x}\right] \times\left[-L_{y}, L_{y}\right] \times\left[-L_{z}, L_{z}\right]$, where the length of domain is $L_{x}=100 r_{a}$, and its width and height in the inlet and outlet sections are $L_{y}=L_{z}=10 r_{a}$ and $L_{y}=L_{z}=40 r_{a}$. The mesh contains $350 \times 150 \times 150$ nodes. In the initial region of the jet, the mesh is uniform up to $x \sim 10 r_{a}$, then mesh is refined, so $\Delta x_{\min }=0.08 r_{a}$ and $\Delta x_{\max }=0.15 r_{a}$. In the cross section, the mesh nodes are concentrated towards the nozzle outlet, so $\Delta y_{\min }=\Delta z_{\min }=0.03 r_{a}$ and $\Delta y_{\max }=\Delta z_{\max }=0.09 r_{a}$. The time step is $\Delta t=0.08 r_{a} / u_{a}=5.8 \times 10^{-5} \mathrm{~s}$. A statistically reliable averaged pattern of flow is obtained by making $10^{5}$ time steps.

Correct specification of boundary conditions requires the calculation of flow in the nozzle 
and boundary layer on the external surface of the nozzle [32] (a part of the nozzle is included in the computational domain). No calculation of flow in nozzle is performed, and a velocity profile is specified at the nozzle outlet boundary at $|r| \leq r_{a}$, on which random sinusoidal oscillations are imposed [33]

$$
v_{x}(r, t)=\frac{u_{a}}{3}\left[1+\tanh \left(\frac{0.5-|r|}{2 \delta}\right)\right][1+\alpha \sin (\operatorname{Sh} t)],
$$

where $\delta$ is the momentum thickness, $\alpha$ is the amplitude of disturbances and Sh is the Strouhal number. It is assumed that $\delta / r_{a} \sim 0.1, \alpha=2.1 \times 10^{-3}$ and $\mathrm{Sh}=0.42$. Small random oscillations are further imposed on the radial distribution of circumferential velocity

$$
v_{\theta}(r, t)=0.025 \exp \left[-3(1-|r|)^{2}\right] \phi,
$$

where $\phi$ is a random number from uniform distribution in $[-0.5,0.5]$. The radial velocity at the nozzle exit is taken to be zero $v_{r}(r, t)=0$.

Large-scale eddy structures present in the shear layer and shown in the Figure 11 have the form of toroidal axially symmetric eddies merging at a certain distance apart from the nozzle outlet (of the order of one to two section diameters). Eddy structures existing in the initial part of the jet are small. The typical eddy size increases from the start point downstream, intensifying the exchange of momentum between the layer and the surrounding fluid. The ellipsoidal shape of the coherent structure points to the anisotropy of turbulent pulsations in the region where large-scale eddies exist.
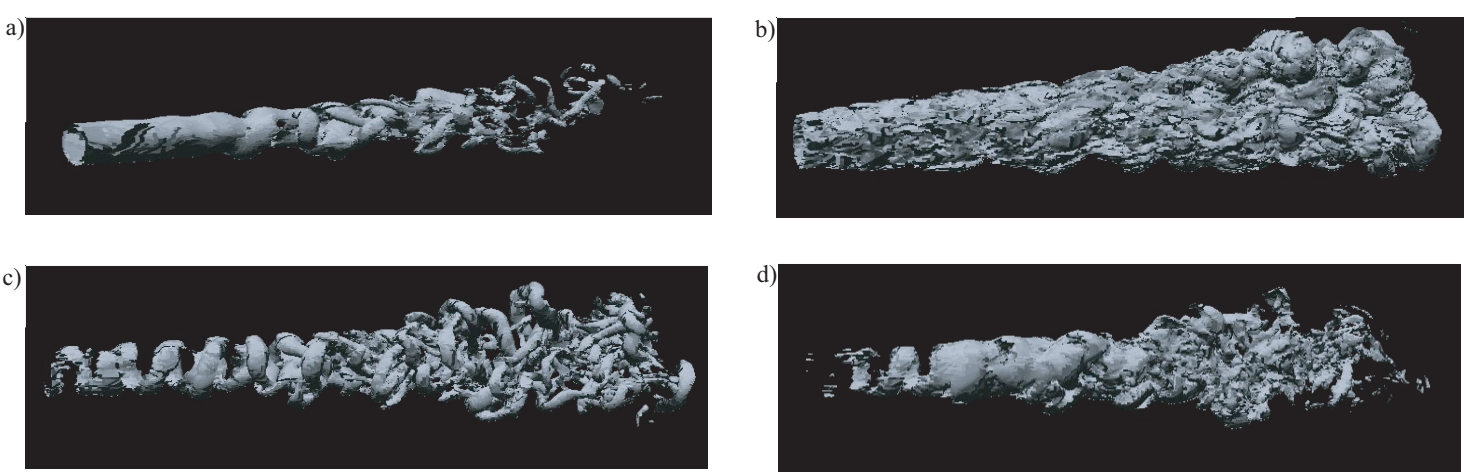

Figure 11. Visualization of flow field in the turbulent jet with the use of contours of vorticity $(a)$, helicity $(b), Q$-criterion $(c)$ and $\lambda_{2}$-criterion $(d)$

The generation of eddies is due to the Kelvin-Helmholtz instability of the shear layer. The maximum and minimum of the vorticity approximately correspond to the eddy centers. At low Reynolds numbers, Re $\sim 10^{3}$, the jet at the nozzle outlet is nearly axially symmetrical. As the Reynolds number increases with distance from the nozzle up to $\operatorname{Re} \sim 10^{4}$, a weak sinusoidal mode appears.

The results computed demonstrate that the velocity and temperature profiles in the flow cross sections exhibit a typical jet pattern, as well as a kink in the middle part. The profiles become wider with increasing axial coordinate, which points to the increase in the thickness of the zone of jet mixing with the surrounding fluid. Near the jet boundary, the profiles become sloping.

As the degree of preheating of the jet increases, the maximum of fluctuations of axial velocity shifts towards the nozzle outlet. A further increase in the degree of preheating causes a qualitative variation of the pattern of this impact, and the maximum of fluctuations of axial velocity decreases. A similar impact is made by the non-isothermality of flow on the 
distribution of intensity of temperature fluctuations, the maximum of which is of the order of $13 \%$ of the temperature difference at the nozzle outlet.

The maximum of fluctuations of axial velocity occurs at $x / r_{a} \sim 16$. In the region of the core of constant velocity, the intensity of turbulence, while remaining almost constant in the transverse direction, increases from $1.5-2 \%$ at the nozzle outlet to a value of the order of $6 \%$ at the end of the initial region with increasing distance from the nozzle. Because no turbulent mixing is present within the jet core, the effect of the increase in the degree of turbulence is associated with the penetration of pressure fluctuations from the mixing zone into the core. The fluctuations of velocity on the jet axis reach a maximum at a distance equal to two lengths of initial region. This value remains almost constant for both isothermal and non-isothermal jets and depends relatively weakly on the conditions of outflow.

The radial coordinate, at which the correlation moments of velocity and temperature reach a maximum, almost coincide in sections $x / r_{a}=10$ and $x / r_{a}=30$, and correspond to the position of maximum of turbulent kinetic energy $\left(r / r_{u}=0.8\right.$ according to the data of [34] and $r / r_{u}=0.7$ according to the data of [35]).

In the near field of jet at $x / r_{a} \sim 10$, the heat transfer occurs along axial coordinate, because $\left\langle v_{x}^{\prime} T^{\prime}\right\rangle /\left\langle v_{r}^{\prime} T^{\prime}\right\rangle \sim 2$ at $0.2<r / r_{u}<1.4$. Downstream at $x / r_{a} \sim 30$, the contributions made by the axial and radial heat fluxes become approximately equal, so that $\left\langle v_{x}^{\prime} T^{\prime}\right\rangle /\left\langle v_{r}^{\prime} T^{\prime}\right\rangle \sim 1.1$ at $0.5<r / r_{u}<1.8$. In a plane jet, $\left\langle v_{x}^{\prime} T^{\prime}\right\rangle /\left\langle v_{r}^{\prime} T^{\prime}\right\rangle \sim 2$ at $x / r_{a}=80$ and $0.4<r / r_{u}<1.3[36]\left(\operatorname{Re}=7.9 \times 10^{3}\right.$ and $\left.\Delta T=25 \mathrm{~K}\right)$. In an axisymmetric jet, the measurements of [36] give $\left\langle v_{x}^{\prime} T^{\prime}\right\rangle /\left\langle v_{r}^{\prime} T^{\prime}\right\rangle \sim 1$ downstream of section $x / r_{a} \sim 100$ and at $0.3<r / r_{u}<1.3$.

The profiles of correlation moments of density and velocity along the jet axis, which characterize the anisotropy of mass flow, are in qualitative agreement with the data of [37] based on $k-\varepsilon$ turbulence model which does not include the anisotropy of turbulent fluctuations of velocity but enables one to estimate the anisotropy of diffusion flow of mass. The anisotropy is observed at $4<x / r_{a}<40$, and $\left\langle\rho^{\prime} v_{r}^{\prime}\right\rangle /\left\langle\rho^{\prime} v_{x}^{\prime}\right\rangle \sim 1$ at $x / r_{a}>40$ downstream.

The spectrum for the jet is steeper than that for the mixing layer (Figure 12) because of a stronger turbulent mixing, and is almost independent on the Reynolds number at high wavenumbers $\left(2 r_{a} k_{x}>1\right.$, where $r_{a}$ is the nozzle-exit radius). The values calculated for the jet are normalized to $\Delta n=\left|n_{a}-n_{\infty}\right|$ and $L=2 r_{a}$, where the subscripts $a$ and $\infty$ refer to the parameters at the nozzle outlet and in submerged space.

\section{Conclusion}

The semi-empirical models of turbulence underpredict the dispersion of fluctuations of the wavefront phase function. Semi-empirical relations derived from the vortex model of the flow ensure reasonably accurate calculations of the root-mean-square values of optical aberrations in the boundary layer on a flat plate and in the free mixing layer. The dependence of the root-mean-square fluctuations of the wavefront phase function on the dynamic pressure is linear for the boundary layer on a flat plate. For the mixing layer, this dependence is linear beginning from the coordinate counted along the flow direction.

The results obtained provide information for designing and applying adaptive-optic equipment and techniques, and they are potentially beneficial in design and improvement of optical systems, where the control of laser beam propagation is an important problem (non-intrusive measurement methods, coherent optical adaptive equipment and techniques) based on the optical detection of light-scattering properties of the flow. 


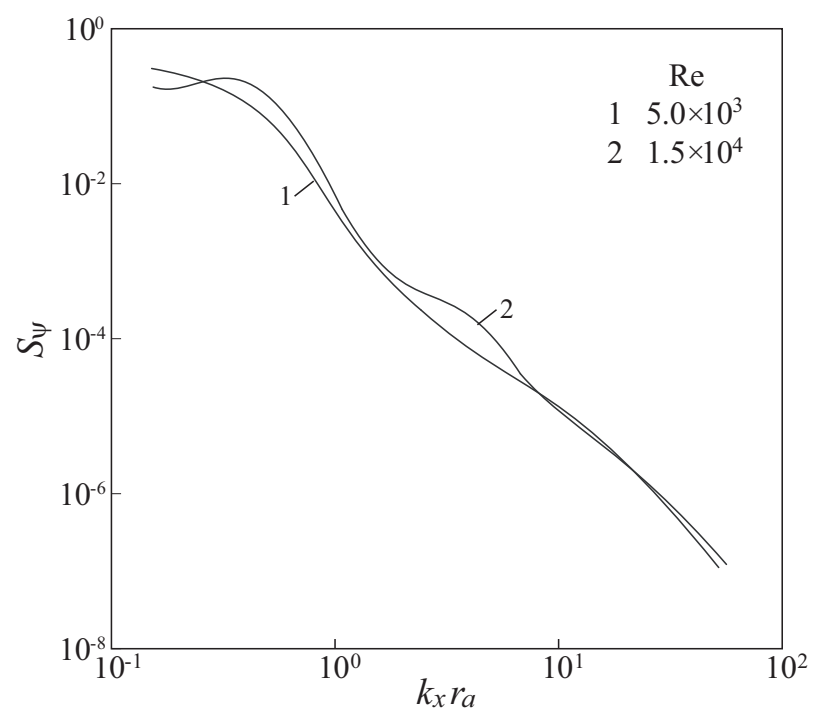

Figure 12. Spectrum of phase fluctuations in the round turbulent jet

\section{Acknowledgements}

The research leading to these results has been partially supported by the European Community's Research Infrastructure Action (grant agreement VISIONAIR 262044) under the 7th Framework Programme (FP7/2007-2013).

\section{References}

[1] Volkov K.N., Emelyanov V.N., Pustovalov A.V. Supersonic flows in channels and jets when applied to the problems of designing aerodynamic windows of gas lasers. Journal of Engineering Physics and Thermophysics, 2015, 88(5), 1210-1220.

[2] Jumper E.J., Fitzgerald E.J. Recent advances in aero-optics. Progress in Aerospace Sciences, 2001, 37(3), 299-339.

[3] Sutton G.W. Aero-optical foundations and applications. AIAA Journal, 1985, 23(10), $1525-1537$.

[4] Sutton G.W. Effect of inhomogeneous turbulence on imaging through turbulent layers. Applied Optics, 1994, 33(18), 3972-3976.

[5] Siegenthaler J.P., Gordeyev S., Jumper E. Shear layers and aperture effects for aerooptics. AIAA Paper, 2005, 2005-4772.

[6] Siegenthaler J.P., Jumper E.J., Gordeyev S. Atmospheric propagation vs. aero-optics. AIAA Paper, 2008, 2008-1076.

[7] Zubair F.R., Catrakis H.J. Aero-optical interactions along laser beam propagation paths in compressible turbulence. AIAA Journal, 2007, 45(7), 1663-1674.

[8] Volkov K.N. Effect of turbulence on propagation of a coherent beam in the boundary layer and mixing layer. Journal of Applied Mechanics and Technical Physics, 2010, 51(6), 827-838.

[9] Dimotakis P.E., Catrakis H.J., Fourguette D.C. Flow structure and optical beam propagation in high-Reynolds number gas-phase shear layer and jets. Journal of Fluid Mechanics, 2001, 433, 105-134. 
[10] Tromeur E., Garnier E., Sagaut P. Analysis of the Sutton model for aero-optical properties of compressible boundary layers. Journal of Fluids Engineering, 2006, 128(2), 239-246.

[11] Truman C.R. The influence of turbulent structure on optical phase distortion through turbulent shear flows. AIAA Paper, 1992, 92-2817.

[12] Zhang Y.-P., Fan Z.-G. Study on the optical path difference of aero-optical window. International Journal for Light and Electron Optics, 2007, 118(12), 557-560.

[13] Wu L., Fang J., Yang Z., Wu S. Study on a neural network model for high speed turbulent boundary layer inducing optical distortions. International Journal for Light and Electron Optics, 2011, 122(17), 1572-1575.

[14] Lifshitz Y., Degani D., Tumin A. On the interaction of turbulent shear layers with harmonic perturbations. Flow, Turbulence and Combustion, 2008, 80(1), 61-80.

[15] Childs R.E. Prediction and control of turbulent aero-optical distortion using large eddy simulation. AIAA Paper, 1993, 93-2670.

[16] Jones M.I., Bender E.E. CFD-based computer simulation of optical turbulence through aircraft flowfields and wakes. AIAA Paper, 2001, 2001-2798.

[17] Gordeyev S., Jumper E. Fluid dynamics and aero-optics of turrets. Progress in Aerospace Sciences, 2010, 46(8), 388-400.

[18] Wang T., Zhao Y., Xu D. Numerical study of evaluation optical quality of supersonic flow fields. Applied Optics, 2007, 46(23), 5545-5551.

[19] Wei H., Chen C.P. A nonequilibrium algebraic model for turbulent density fluctuations. International Journal of Heat and Mass Transfer, 1996, 39(18), 3989-3991.

[20] Huang P.G., Coleman G.N., Bradshaw P. Compressible turbulent channel flows: DNS results and modeling. Journal of Fluid Mechanics, 1995, 305, 185-218.

[21] Volkov K. Numerical analysis of Navier-Stokes equations on unstructured meshes / Handbook on Navier-Stokes equations: theory and analysis. Nova Science, 2016, 365442.

[22] Smirnov N.N., Betelin V.B., Nikitin, V.F., Stamov, L.I., Altoukhov D.I. Accumulation of errors in numerical simulations of chemically reacting gas dynamics. Acta Astronautica, 2015, 117(1), 338-355.

[23] Emelyanov V.N., Karpenko A.G., Kozelkov A.S., Teterina I.V., Volkov K.N., Yalozo A.V. Analysis of impact of general-purpose graphics processor units in supersonic flow modelling. Acta Astronautica, 2017, 135, 198-207.

[24] Schlichting H., Gersten K. Boundary layer theory. Springer Verlag, Berlin, 2000.

[25] Gordeyev S., Jumper E.J. Aero-optical characteristics of compressible, subsonic turbulent boundary layers. AIAA Paper, 2003, 2003-3606.

[26] Fitzgerald E.J., Jumper E.J. Scaling aero-optic aberrations produced by high-subsonicMach shear layer. AIAA Journal, 2002, 40(7), 1373-1381.

[27] Gilbert K.G. KC-135 aero-optical boundary-layer/shear-layer experiments / AeroOptical Phenomena. New York, 1982, 80, 306-324.

[28] Li Q., Fu S. Numerical simulation of high-speed planar mixing layer. Computers and Fluids, 2003, 32(10), 1357-1377.

[29] Papamoschou D., Roshko A. The compressible turbulent shear layer: an experimental study. Journal of Fluid Mechanics, 1988, 197, 453-477.

[30] Elliott G.S., Samimy M. Compressibility effects in free shear layers. Physics of Fluids, 1990, 2(7), 1231-1240. 
[31] Goebel S.G., Dutton J.C. Experimental study of compressible turbulent mixing layers. AIAA Journal, 1991, 29(4), 538-546.

[32] Tucker P.G. Novel MILES computations for jet flows and noise. International Journal of Heat and Fluid Flow, 2004, 25(4), 625-635.

[33] Boersma B.J., Lele S.K. Large eddy simulation of compressible turbulent jets / Annual Research Brief. Center for Turbulence Research, 1999, 365-377.

[34] Chevray R., Tutu N.K. Intermittency and preferential transport of heat in a round jet. Journal of Fluid Mechanics, 1978, 88, 133-160.

[35] Chua L.P., Antonia R.A. Turbulent Prandtl number in a circular jet. International Journal of Heat and Mass Transfer, 1990, 33(2), 331-339.

[36] Antonia R.A., Prabhu A., Stephenson S.E. Conditionally sampled measurements in a heated turbulent jet. Journal of Fluid Mechanics, 1975, 72, 455-480.

[37] Gazzah M.H., Sassi M., Sarh B., Gokalp I. Numerical simulation of variable density subsonic turbulent jets by using the $k-\varepsilon$ model. International Journal of Thermal Sciences, 2002, 41(1), 51-62. 